\title{
Acolhimento com classificação de risco em unidades de pronto atendimento: avaliação sob a ótica do enfermeiro
}

\author{
Embracement with risk classification in residential care units: \\ evaluation from nurse's viewpoint
}

Mayzza Campina RoDrigues ${ }^{I}$ ALyne Nunes Mota ${ }^{1}$ Rebeca Eloy de Melo ${ }^{\text {II }}$ Eduardo Espíndola Sousa ${ }^{\mathrm{I}}$ Fernando Rodrigues Peixoto QUARESMA $^{\text {II }}$

Erika da Silva Maciel ${ }^{\text {II }}$

${ }^{\mathrm{I}}$ Enfermeira(o). Palmas/TO - Brasil "Universidade Federal do Tocantins (UFT). Palmas/TO - Brasil
Resumo Objetivo: O objetivo do trabalho foi avaliar a percepção dos enfermeiros frente ao Acolhimento com Classificação de Risco implantado em Unidades de Pronto Atendimento (UPA). Método: Trata-se de um estudo descritivo de caráter corte transversal e abordagem quantitativa realizado, em 2015, com a aplicação de um instrumento tipo questionário, direcionado aos enfermeiros das UPAs. Para a análise dos dados, utilizou-se estatística padrão e a classificação se deu conforme os escores preconizados na metodologia dos instrumentos. Resultados: Participaram do estudo 35 profissionais. De acordo com suas respostas sobre o acolhimento no setor de Classificação de Risco, dimensões de Estrutura, Processo e Resultados, este foi classificado de forma geral como precário. Conclusão: Os resultados indicam que os serviços oferecidos demandam ações para o aprimoramento da Política Nacional de Humanização. A capacitação no acolhimento à demanda espontânea pode representar um caminho para melhoria das condições dos serviços de saúde ofertados.

Palavras-chave: Humanização. Acolhimento. Enfermeiro.

Abstract Objective: The objective of the study was to assess the nurses' perception of the Reception with Risk Classification implemented in Emergency Care Units (UPA). Method: This is a descriptive study with a cross-sectional character and a quantitative approach conducted in 2015, with the application of a questionnaire-type instrument, aimed at nurses from the UPAs. For data analysis, standard statistics were used and the classification was according to scores recommended in the methodology of the instruments. Results: 35 professionals participated in the study, who, according to their answers about the reception in the risk rating sector, the immensities of Structure, Process and Results, were generally classified as Regular. Conclusion: The results indicate that the services offered demand actions to improve the National Humanization Policy. Training in welcoming spontaneous demand can represent a way to improve the conditions of the health services offered.

Keywords: Humanization. Reception. Nurse. 


\section{INTRODUÇÃO}

No ano de 2006, o Ministério da Saúde (MS) regulamentou a estratégia Acolhimento com Classificação de Risco (ACCR), tendo como referencial as diretrizes da Política Nacional de Humanização (PNH). O principal objetivo do ACCR é humanizar as ações voltadas para o cuidado aos usuários nos serviços de urgência e emergência. ${ }^{1}$

Entende-se o ACCR como um mecanismo de humanização ao atendimento, que visa estabelecer mudanças na forma de atendimento dos usuários, sendo capaz de acolher o cidadão e garantir a ele um melhor acesso aos serviços de urgência e emergência. ${ }^{2}$

Para que isso seja possível, é necessário que o profissional ofereça uma escuta qualificada aos usuários, sendo capaz de classificar, mediante protocolo, as queixas desses pacientes, a fim de encaminhá-los a um atendimento ideal de acordo com as necessidades de suas demandas. $\mathrm{O}$ atendimento da população com queixas que não correspondem a situações de emergência sobrecarrega os serviços de urgência e emergência com o desenvolvimento de ações assistenciais que poderiam ser realizadas em unidades de atenção primária à saúde. ${ }^{3}$

Assim, a superlotação tem se tornado recorrente nos serviços de urgência e emergência em todo o país, tanto nas Unidades de Pronto Atendimento (UPA) quanto nos Serviços Hospitalares de Emergência (SHE), aspecto que pode estar relacionado ao perfil demográfico da população, alta demanda espontânea e a pouca efetividade dos serviços de prevenção em saúde. ${ }^{1,2,3,4}$

Foi justamente diante desse cenário desafiador que o Ministério da Saúde (MS), por meio da Política Nacional de Humanização (PNH), teve a intenção de reorganizar os serviços de emergência e propôs a im- plantação do Acolhimento com Classificação de Risco (ACCR).

Entretanto, classificar o risco de pacientes, além de desafiador, exige do profissional competências, e habilidades desenvolvidas para o desempenho satisfatório. Entre os principais atributos necessários aos profissionais destaca-se a oferta de uma escuta qualificada aos usuários, que seja capaz de classificar, mediante protocolo definido e que proporcione respaldo técnico científico, as queixas de saúde desses pacientes, conforme seus sinais e sintomas apresentados, encaminhando-os ao atendimento, segundo as necessidades de suas demandas. ${ }^{5}$

Os protocolos a serem seguidos nos serviços de urgência e emergência encontram melhores resultados quando atendem a PNH e as diretrizes de ACCR, e podem ser protocolos definidos pelos serviços de saúde ou protocolos internacionais. ${ }^{6}$

Entre os protocolos internacionais tem-se o protocolo de Manchester, que tem sido amplamente utilizado nos serviços de urgência brasileiros. ${ }^{3}$

Nesse contexto, o enfermeiro participa ativamente no processo de classificação de risco nas UPAs, cumprindo seu papel legal durante a consulta de enfermagem de atender aos pacientes por riscos clínicos e contribuiu com a regulação de atendimento em uma das principais portas de entrada dos serviços de emergência., ${ }^{3,4}$

O ACCR, quando realizado de forma humanizada, melhora a eficiência e eficácia assim como reduz as iniquidades nos serviços de saúde. Diante do exposto, como os enfermeiros das Unidades de Pronto Atendimento avaliam o ACCR dentro da instituição?

Esta pesquisa tem como objetivo avaliar a percepção dos Enfermeiros das Unidades de Pronto Atendimento de Palmas/TO, frente ao Acolhimento e Classificação de Risco - 
ACCR, por eles realizados, com base nos objetivos da Política Nacional de Humanização propostos pelo Ministério da Saúde.

\section{Métodos}

Trata-se de um estudo descritivo de caráter transversal e abordagem quantitativa, realizado, em março de 2015, na capital do Tocantins/Palmas, desenvolvido nas duas únicas Unidades de Pronto Atendimento (UPA) do município de Palmas - TO, em março de 2015. Essas unidades utilizam um protocolo de classificação de risco com base nas recomendações de Bellucci Júnior. ${ }^{7}$

Os critérios de inclusão para participação do estudo foram: ser enfermeiro concursado ou contratado e concordar em participar da pesquisa de forma voluntária por meio da assinatura do Termo de Consentimento Livre e Esclarecido TCLE). Os critérios de exclusão foram estar de férias ou licença por qualquer motivo e participantes que deixaram de responder a mais de $20 \%$ do total de questões do instrumento para a Avaliação do ACCR.

Para a coleta de dados, foram utilizados os seguintes instrumentos: questionário socioeconômico-demográfico elaborado pelos pesquisadores com informações como: sexo, idade, tempo de serviço, regime de trabalho, tempo de descanso/repouso durante jornada, quantidade de locais de trabalho, renda e o instrumento para a Avaliação do Acolhimento com Classificação de Risco proposto por Bellucci Júnior, ${ }^{7}$ composto por 21 questões, dispostas na forma de escala do tipo Likert, com valores que variam de "Discordo totalmente" (valor 1) a "Concordo totalmente" (valor 5). Entre as 21 questões, 12 têm sentido positivo e nove, negativo. Essas questões estão assim distribuídas no instrumento: avaliação da dimen- são Estrutura = questões de $1 \mathrm{a} 7$; avaliação da dimensão Processo = questões de 8 a 14; e avaliação da dimensão Resultado $=$ questões de 15 a 20.

Cada dimensão aborda itens diferentes, sendo a dimensão Estrutura voltada para atributos da instalação onde é prestado o atendimento, incluindo os recursos humanos, materiais, financeiros e a estrutura organizacional. Já a dimensão Processo diz respeito às atividades realizadas para a prestação do atendimento e as relações estabelecidas entre os profissionais e usuários. Por último, temos a dimensão Resultado, que são os efeitos na saúde e as mudanças de comportamento dos usuários a partir do conhecimento adquirido. ${ }^{7}$

As entrevistas foram realizadas por equipe de quatro pesquisadores, previamente treinada durante os turnos de serviço de forma individualizada. Os dados foram tabulados em planilha do software Microsoft Excel versão XP, 2007, e para a análise utilizou-se estatística descritiva por meio de distribuição em frequência e percentuais. A classificação se deu conforme os escores preconizados na metodologia do instrumento Avaliação do Acolhimento com Classificação de Risco, que recomenda: para analisar os dados do escore geral, fez-se a soma dos resultados de cada dimensão e se dividiu pela quantidade de indivíduos que participaram da pesquisa. $\mathrm{O}$ mesmo procedimento é proposto para a avaliação de cada dimensão, calculada a média dos valores de cada item, dividido pela quantidade de indivíduos, que participaram da pesquisa e assim comparado aos dados, após inversão das afirmações (itens $3,4,5$, $7,10,14,16,19$ e 20) que correspondiam à forma negativa na escala. Foi calculada a média ponderada (MP) da pontuação atribuída pelo número de respondentes (NR) a cada item, da seguinte forma: 
$\mathrm{MP}=\quad(1 \times \mathrm{NR})+(2 \times \mathrm{NR})+(3 \times \mathrm{NR})$ $+(4 \times \mathrm{NR})+(5 \times \mathrm{NR})$

Total de respondentes

Para a avaliação das dimensões e do ACCR, verificaram-se os escores com base nos seguintes parâmetros: ${ }^{7}$

Ótimo = 31,5 a 35 pontos;

Satisfatório = 26,2 a 31,4 pontos;

Precário = 17,5 a 26,1 pontos; e

Insuficiente $=7$ a 17,4 pontos

Para a Avaliação Geral do ACCR, utilizaram-se os seguintes parâmetros: ${ }^{7}$

Ótimo = 94,5 a 105 pontos;

Satisfatório = 78,7 a 94,4 pontos;

Precário $=52,5$ a 78,6 pontos e;

Insuficiente $=21$ a 52,4 pontos.

O presente trabalho foi submetido e aprovado pelo Comitê de Ética em Pesquisas com Seres Humanos (CEP) via plataforma Brasil, sob o número 912.537, como também pela a Secretaria Municipal de Saúde (SEMUS) de Palmas - Tocantins.

\section{RESULTADOS}

Um total de 44 enfermeiros foi recrutado e avaliado para a elegibilidade do estudo. Três enfermeiros estavam em período de férias e seis se recusaram participar do estudo. Dessa forma, completaram o estudo um total de 35 enfermeiros ( $80 \%$ do total).

Entre os resultados socioeconômico-demográfico, destaca-se que 27 (77 \%) foram profissionais do sexo feminino. A média de idade da amostra foi de 38,08 $( \pm 9,79)$ anos. Observou-se que $23(65,3 \%)$ possuem pelo menos dois vínculos empregatícios (Tabela 1).
Tabela 1 - Características socioeconômica-demográficas dos profissionais da Unidade de Pronto Atendimento em Palmas/ TO, 2016.

\begin{tabular}{|c|c|}
\hline Variáveis & Média (DP) /n (\%) \\
\hline \multicolumn{2}{|l|}{ Sexo } \\
\hline Masculino & $8(23 \%)$ \\
\hline Feminino & $27(77 \%)$ \\
\hline Média de idade & $38,08( \pm 9,79)$ \\
\hline $\begin{array}{c}\text { Vínculo Empregatício } \\
\text { Concursado }\end{array}$ & $33(94,3 \%)$ \\
\hline Contratado & $2(5,7 \%)$ \\
\hline \multicolumn{2}{|l|}{ Regime de Trabalho } \\
\hline 6 a 8 horas & $2(5,7 \%)$ \\
\hline $\begin{array}{l}10 \text { a } 12 \text { horas } \\
\text { Mais de } 12 \text { horas }\end{array}$ & $\begin{array}{l}9(25,7 \%) \\
24(68,6 \%)\end{array}$ \\
\hline \multicolumn{2}{|l|}{ Tempo de Serviço } \\
\hline$<1$ ano & $17(48,6 \%)$ \\
\hline $\begin{array}{l}1 \text { a } 5 \text { anos } \\
6 \text { a } 10 \text { anos } \\
+ \text { de } 10 \text { anos } \\
\end{array}$ & $\begin{array}{l}3(8,6 \%) \\
10(28,6 \%) \\
5(14,3 \%) \\
\end{array}$ \\
\hline \multicolumn{2}{|l|}{ Descanso e Repouso } \\
\hline Não tem & $1(2,9 \%)$ \\
\hline Uma hora & $14(40,0 \%)$ \\
\hline Duas horas & $20(57,1 \%)$ \\
\hline $\begin{array}{l}\text { Trabalha em quantos } \\
\text { locais } \\
\qquad \begin{array}{c}1 \\
2\end{array}\end{array}$ & $\begin{array}{l}9(25,7 \%) \\
23(65,3 \%)\end{array}$ \\
\hline 3 & $3(8,6 \%)$ \\
\hline \multicolumn{2}{|l|}{ Renda Mensal Total } \\
\hline $\begin{array}{ll} & \text { Não contribuído } \\
& \mathrm{R} \$ 790,00 \text { a R\$ } \\
5.000,00 & \\
\end{array}$ & $\begin{array}{l}2(5,7 \%) \\
12(34,3 \%)\end{array}$ \\
\hline $9.000,00 \quad \mathrm{R} \$ 6,000,00$ a R\$ & $\begin{array}{l}14(40,0 \%) \\
5(14,3 \%)\end{array}$ \\
\hline $\begin{array}{c}\mathrm{R} \$ 10.000,00 \mathrm{a} \\
>\mathrm{R} \$ 20.000,00\end{array}$ & $2(5,7 \%)$ \\
\hline
\end{tabular}


Os resultados da avaliação do instrumento de ACCR indicaram um escore geral de 64,97, o que a classifica como precária. Quando calculado os resultados obtidos em cada dimensão Donabediana: estrutura, processo e resultado, a pontuação alcançada também foi classificada como precária, conforme padronização do instrumento (Tabela 2).

Tabela 2 - Classificação do instrumento de ACCR frente à percepção dos profissionais das Unidades de Pronto Atendimento em Palmas/TO, 2015.

\begin{tabular}{lll}
\hline Dimensão & Pontuação & Classificação \\
\hline Estrutura & 22,31 & Precário \\
Processo & 24,04 & Precário \\
Resultado & 18,60 & Precário \\
Escore geral & 64,97 & Precário \\
\hline
\end{tabular}

\section{DIscussão}

Os profissionais de enfermagem das UPAs, em sua maioria, são do sexo feminino com idade produtiva, o que converge com resultados semelhantes, que mostram a predominância do gênero nessa profissão. ${ }^{8}$ Destaca-se ainda que a faixa etária média de 38 anos seja característica para atender às exigências impostas pelo serviço, que demanda de disposição para a realização das atividades. ${ }^{8-9)}$

Em relação ao tempo de serviço, os resultados indicam que parte considerável $(28,6 \%)$ atua entre 6 a 10 anos na área, logo o ACCR faz parte da rotina dos profissionais há anos.
Entretanto, no presente estudo a avaliação do ACCR, conforme o instrumento proposto por Bellucci, ${ }^{7}$ obteve classificação precária para os escores das dimensões Estrutura, Processo e Resultado. Esses resultados corroboram com outros estudos realizados no Brasil. ${ }^{10-11}$

Em estudo realizado na UPA com a participação de equipe multidisciplinar em Mossoró/RN a classificação das dimensões foi similar à encontrada em nosso estudo. Tais resultados para a dimensão Estrutura indicam a deficiência de sinalização e falta de estrutura física nos serviços de Urgência e Emergência e divergem dos conceitos propostos pela $\mathrm{PNH}$, onde a adequação de condições físicas deve ser assegurada com o intuito de acolher e acomodar os usuários e seus acompanhantes. ${ }^{11}$

A partir desses resultados, compreende-se a necessidade de melhorias no processo de atendimento dos serviços de Urgência e Emergência. As falhas nas pactuações internas e externas aos processos de trabalho e nos serviços prestados refletem a precarização dos serviços prestados. ${ }^{8,12,13}$

A dimensão Processo obteve a classificação precária, que pode se justificar pela falta de conhecimento e domínio sobre a $\mathrm{PNH}$, a escassez de capacitações da equipe atuante e falta de comunicação entre equipe e usuário. As dificuldades no atendimento aos usuários, que passam pelo ACCR, geram insegurança em ambas as partes, afetam a melhoria da qualidade do atendimento, e contradizem a $\mathrm{PNH}$, no que se refere ao respeito do fluxo de atendimento aos usuários e ao conhecimento das condutas do ACCR, a fim de propiciar segurança aos mesmos e estabelecer relações entre profissionais e usuários. ${ }^{9}$ 
As dificuldades encontradas também estão relacionadas ao espaço físico de trabalho, que é insuficiente, e ausência de materiais, problemas esses que se agravam com o constante acúmulo de usuários. ${ }^{15}$

A avaliação geral do ACCR e as dimensões avaliadas no instrumento foram classificadas como precárias. Esse achado sugere que o conceito de Humanização não está sendo aplicado em todos os momentos durante o atendimento ao usuário.

Porém, os resultados devem ser analisados com cautela, pois tem-se como limitação do estudo o tamanho da amostra reduzido, provocado por recusas em participação, férias ou licenças dos profissionais.

\section{CONSIDERAÇões FINAIS}

Diante do que preconiza a PNH criada e difundida pelo Ministério da Saúde, os achados desta pesquisa classificam os setores de acolhimento das UPAs de Palmas/Tocantins como precários.

Sugere-se como oportunidades de melhorias a sinalização do ambiente, conforto aos usuários e funcionamento dos mecanismos de contra referência. Além de capacitação com enfermeiros para construção e fortalecimento da autonomia, para que possam proporcionar um acolhimento mais humanizado e um atendimento individualizado mais eficaz.

\section{REFERÊNCIAS}

1. Oliveira T. D. A., Pinto K. A. Acolhimento com classificação de risco e acesso em serviço de emergência: avaliação de usuários. Ciência, Cuid e Saúde, v. 14, n. 2, abr./jun. 2015.

2. Inoue K. C., Murassaki A. C. Y., Bellucci Júnior J. A., Rossi RM, Martinez Y., Matsuda LM. Acolhimento com classificação de risco: avaliação da estrutura, processo e resultado. Revista Mineira de Enfermagem, v. 19, n. 1, p. 13-28, 2015.

3. Diniz A. S., Silva A. P. da, Souza C. C. de, Chianca T. C. M. Demanda clínica de uma unidade de pronto atendimento, segundo o protocolo de Manchester. Revista Eletrônica de Enfermagem, v. 16, n. 2, p. 312-20, 2014.

4. Silva P. L., Paiva L., Faria V. B., Ohl R. I. B, Chavaglia S. R. R. Acolhimento com classificação de risco do serviço de Pronto-Socorro Adulto: satisfação do usuário. Revista da Escola de Enfermagem da USP, v. 50, n. 3, 2016.

5. Brasil. Ministério da Saúde. Secretaria de Atenção à Saúde. Departamento de Atenção Especializada. Manual instrutivo da Rede de Atenção às Urgências e Emergências no Sistema Único de Saúde (SUS). Brasília: Editora do Ministério da Saúde, 2013, 84 p.: il.

6. Brasil. Ministério da Saúde. Secretaria de Atenção à Saúde. Política Nacional de Humanização da Atenção e Gestão do SUS. Acolhimento e classificação de risco nos serviços de urgência Brasília: Editora do Ministério da Saúde, 2009, 56 p.

7. Bellucci Júnior J. A., Matsuda L. M. Construção e validação de instrumento para avaliação do Acolhimento com Classificação de Risco. Revista Brasileira de Enfermagem, v. 65, n. 5, 2012.

8. Lima Neto AV de, Nunes VM de A., Fernandes RL, Barbosa IML, Carvalho GRP de. Acolhimento e humanização da assistência em pronto-socorro adulto: percepções de enfermeiros. Revista de Enfermagem da UFSM, v. 3, n. 2, p. 276-286, 2013.

9. Versa G. L. G da S., Vituri D. W., Buriola A. A., Oliveira C. A. de, Matsuda L. M. Avaliação do acolhimento com classificação de risco em serviços de emergência hospitalar. Revista Gaúcha de Enfermagem, v. 35, n. 3, p. 21-28, 2014. 
10. Vituri, D. W., Inoue, K. C., Bellucci Júnior, J. A., Oliveira, C. A., Rossi, R. M., Matsuda, L. M. Acolhimento com classificação de risco em hospitais de ensino: avaliação da estrutura, processo e resultado. Revista Latino-Americana de Enfermagem, v. 21, n. 5, 2013.

11. Oliveira K. K. D. de, Amorim K. K. P. S., Fernandes A. P. N. de L., Monteiro A. I. Impacto da implementação do acolhimento com classificação de risco para o trabalho dos profissionais de uma unidade de pronto atendimento. Revista Mineira de Enfermagem, v. 17, n. 1, p. 149-165, 2013.

12. Belucci Júnior J. A., Matsuda L. M. Acolhimento com classificação de risco em serviço hospitalar de emergência: avaliação da equipe de enfermagem. Revista Mineira de Enfermagem, v. 16, n. 3, p. 419-428, 2012.

13. Dal Pai D., Lautert L. Sofrimento no trabalho de enfermagem: reflexos do discurso vazio no acolhimento com classificação de risco. Escola Anna Nery: Revista de Enfermagem. Rio de Janeiro, v. 15, n. 3, p. 524-530, 2011.

14. SALES ZEM, K. K.; MONTEZELI, J. H.; PERES, A. M. Acolhimento com classificação de risco: concepção de enfermeiros de um pronto socorro. Revista da Rede de Enfermagem do Nordeste, v. 13, n. 4, 2012.

15. Nascimento E.R.P do, Hilsendeger B.R., Neth C., Belaver G.M, Bertoncello K.C.G. Classificação de risco na emergência: avaliação da equipe de enfermagem. Rev. enferm. UERJ, p. 84-88, 2011.

\section{DAdOS DOS AUTORES}

Mestre Mayzza Campina Rodrigues.

Enfermeira. Palmas/TO - Brasil. mayzza.rodrigues@gmail.com

Mestre Alyne Nunes Mota.

Enfermeira. Palmas/TO - Brasil. alynenmota@gmail.com

\section{Rebeca Eloy de Melo.}

Graduada em enfermagem pela Universidade Federal do Tocantins (UFT). Palmas/TO - Brasil. rebecaeloy@uft.edu.br

\section{EduARdo Espíndola Sousa.}

Residente do Plano Integrado de Residências em Saúde (PIRS). Fundação Escola de Saúde Pública de Palmas (FESP/PALMAS-TO). Enfermeiro. Palmas/TO - Brasil. eduardoespindolasousa@gmail.com

\section{Prof. Dr.Fernando Rodrigues Peixoto Quaresma.}

Docente da Universidade Federal do Tocantins (UFT). Palmas/TO - Brasil. quaresma@uft.edu.br

\section{Profa.Dra.Erika da Silva Maciel.}

Docente da Universidade Federal do Tocantins (UFT). Palmas/TO - Brasil. erikasmaciel@uft.edu.br

Submetido em: 26-12-2018

Aceito: 22-6-2020 